\title{
1994012388
}

\section{STOCHASTIC DAMAGE EVOLUTION IN TEXTILE LAMINATES}

\author{
Yuris A. Dzenis \\ N $94=16-861$ \\ Center for Composite Materials \\ Department of Mechanical and Aerospace Engineering \\ University of Texas at Arlington \\ Arlington, TX 76019
}

Alexander E. Bogdanovich and Christopher M. Pastore

College of Textiles

North Carolina State University

Raleigh, NC 27695-8301

\begin{abstract}
SUMMARY
A probabilistic model utilizing random material characteristics to predict damage evolution in textile laminates is presented in this paper. Model is based on a division of each ply into two sublaminas consisting of cells. The probability of cell failure is calculated using stochastic function theory and maximal strain failure criterion. Three modes of failure, i.e. fiber breakage, matrix failure in transverse direction, as well as matrix or interface shear cracking are taken into account. Computed failure probabilities are utilized in reducing cell stiffness based on the mesovolume concept. A numerical algorithm is developed predicting the damage evolution and deformation history of textile laminates. Effect of scatter of fiber orientation on cell properties is discussed. Weave influence on damage accumulation is illustrated with the help of an example of a Kevlar/epoxy laminate.
\end{abstract}

\section{INTRODUCTION}

Textile reinforced composites represent an important class of advanced composite materials. Although their structural performance under uniaxial loading yields to that of unidirectional composites, better impact resistance and balanced in-plane properties of these materials as well as ease of application and the resulting low manufacturing cost make them superior for a variety of applications [1]. Ishikava and Chou [1-3] have developed several micromechanical models for textile laminates. Unidirectional mosaic and fiber undulation models are used for effective thermoelastic properties calculation of plain weave fabric composites. The bridging model is developed to simulate the load transfer between the interlaced regions in satin weave composites. Although the elements of stress-strain analysis can be incorporated in the models and effects like "knee behaviour" of textile composites can be described [2], these models do not allow to analyze the damage evolution in textile laminates.

It is well-known that texiile composites under loading undergo multiple damage formation on several structural levels that corresponds to their complex structural hierarchy. The process of damage accumulation in these materials is of stochastic nature due to a number of random factors, such as inevitable scatters in stiffness and strength properties of components, local structural inhomogeneities, and random loading. A combined effect of the above mentioned 
factors causes scatter in static and dynamic strength of finite size specimens. On the other hand, for large specimens, where ali the combinations of random factors are present, their effect may result in gradual accumulation of damage and a definite failure strength. In general, failure analysis of textile composites should involve consideration of several consecutive geometrical models, corresponding to micro-, meso-, and macrolevels of composite structure [4].

Numerous scientists have studied composite strength problems with the help of statistical modeling (see [5-7] and references therein). Ovchinskii [5] proposed and worked out the fundamental principles, models, andalgorithms for stochastic simulation of fracture processes in reinforced composites, starting with micro-level consideration. However, the simulation procedures with micro-level consideration require extensive computational resources for laminate analysis.

A statistical method for reliability analysis of laminates was developed in [8-10]. In these papers, theory of random scalar and vector field excursions was applied for computing the probability of excursions of a random stress-strain field beyond the limiting surface. The method was applied in [11-13] for ply-by-ply stochastic failure analysis and reliability calculation of laminated composite plates and shells. In these papers the whole composite layer was considered as a basic element and maximum probability criterion was used for determination of damage sequence.

Another approach for damage analysis of laminates was developed in [14,15]. The model is based on a division of each layer into statistically large number of mesovolumes. Concentration of broken mesovolumes in plies is calculated as a probability of ply random strains to exceed the limiting failure strains. These concentrations are utilized in ply stiffness reduction. The model allows to predict the gradual damage accumulation and deformation history of composite laminates.

At present, paper probabilistic modeling of damage evolution in textile laminates is performed based on the approach developed in $[14,15]$.

\section{PROBLEM FORMULATION}

Consider a textile reinforced laminated composite sketched in Figure 1,a. Each ply of this laminate consists of two sublaminas, made of a set of cells of two types (Figure 1,b). The orientation of fibers in cell with respect to laminate axes, referred further as cell orientation, is described by angle $\theta$. The relative orientation of cells within the same lamina is not necessarily perpendicular, in general. The weave type in this model is described by fraction of cells of particular orientation in sublamina $v$. The fraction of cells of the other orientation is, obviously, $1-v$. Material properties of cells of different orientations within the same lamina can also be different, in general.

Now, the problem can bis formulated as follows. Assume that initial elastic material properties of cells are random $\tilde{E}^{k}, \tilde{E}_{2_{0}}^{k}, \tilde{G}_{12_{0}}^{k}, \tilde{v}_{12_{0}}^{k}$. Index $\mathrm{k}$ is the cell identification number. Here and further tilda sign $\sim$, upper bar -, and D denote random characteristic, mathematical mean, and dispersion of characteristic, respectively. Load increment applied to such a laminate results in random stress-strain field in each ply, sublamina, and, consequently, in each cell. Even at very low levels of applied load a nonzero probability of cell failure exists and damages start to accumulate in composite. Accumulation of damages causes reduction in laminate stiffness and redistribution of stresses between cells and plies. 
a)
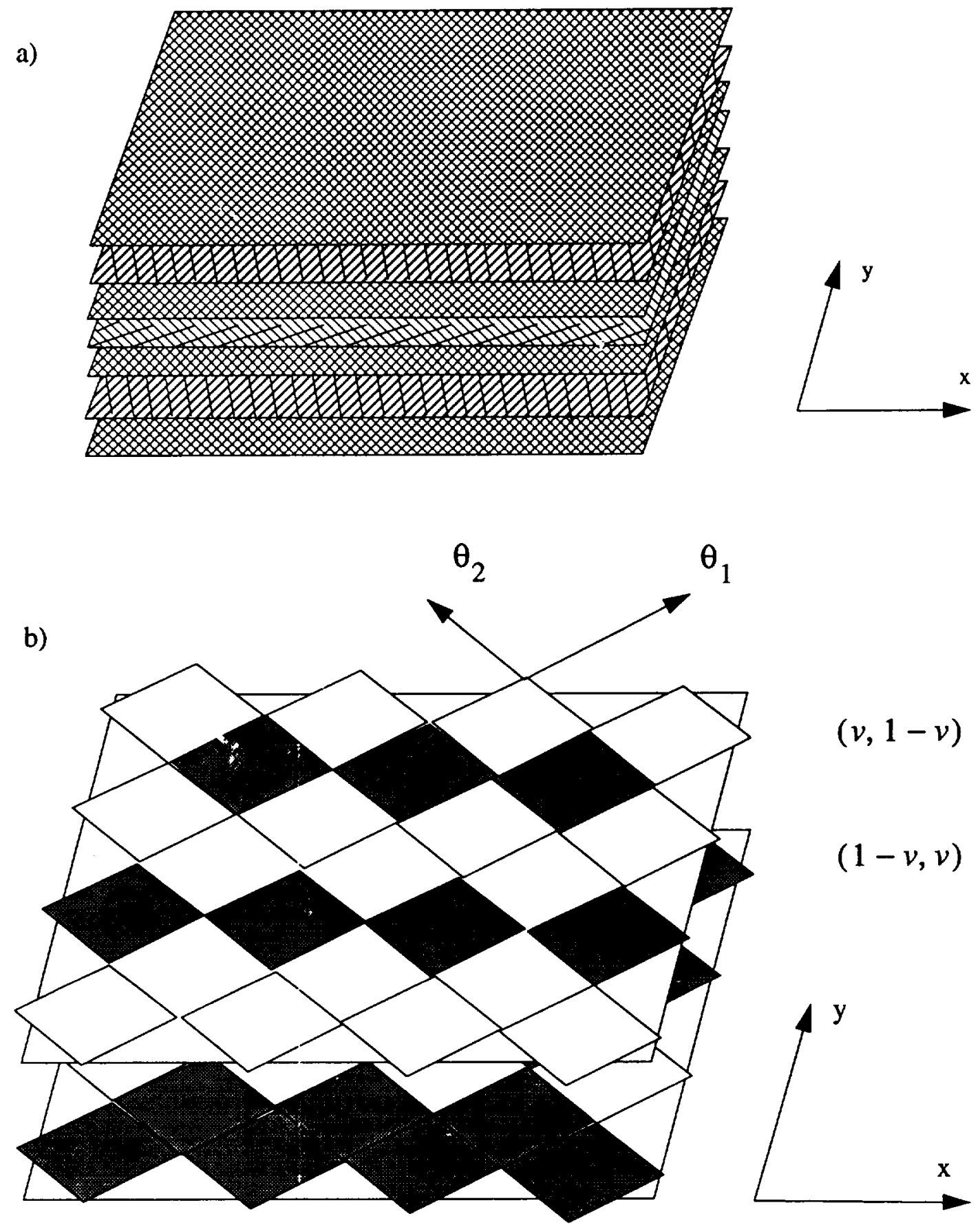

FIGURE 1. Schematic representation of textile laminate (a) and lamina subdivision (b). 
Assume that in-plane stresses applied to composite are monotonically increasing functions of a parameter $t, \sigma_{i}(t)=\left[\sigma_{11}(t), \sigma_{22}(t), \tau_{12}(t)\right]$, with parametric derivatives $\dot{\sigma}_{i}(t)$. Random deformations of composite can then be calculated using integral equations

$$
\bar{\varepsilon}_{i}(t)=\int_{0}^{t} \tilde{S}_{i j}(\tau) \dot{\sigma}_{j}(\tau) d \tau
$$

where $\tilde{S}_{i j}$ are current effective laminate compliances. They depend on cell current elastic properties, ply weave and composite lay-up.

$$
\tilde{S}_{i j}(\tau)=L\left[\tilde{E}_{1}^{k}(\tau), \tilde{E}_{2}^{k}(\tau), \tilde{G}_{12}^{k}(\tau), \tilde{v}_{12}^{k}(\tau), \theta^{k}, v^{k}\right]
$$

The current elastic constants are functions of the initial elastic constants and current damage functions in cells.

$$
\begin{gathered}
\tilde{E}_{1}^{k}(\tau), \tilde{E}_{2}^{k}(\tau), \tilde{G}_{12}^{k}(\tau), \tilde{v}_{12}^{k}(\tau)= \\
M\left[\tilde{E}_{10}^{k}, \tilde{E}_{20}^{k}, \tilde{G}_{12}^{k}, \tilde{v}_{12}^{k}, r_{i}^{k}(\tau)\right]
\end{gathered}
$$

Damage functions may 'se calculated using cell random stress-strain field parameters and some appropriate failure criteria.

$$
r_{i}^{k}(\tau)=R\left[\tilde{\varepsilon}_{i}^{k}(\tau), \tilde{\sigma}_{i}^{k}(\tau)\right]
$$

Cell stress-strain field parameters are calculated, in turn, from known composite strains $\tilde{\varepsilon}_{i}$ (eq. 1).

$$
\tilde{\varepsilon}_{i}^{k}(\tau), \tilde{\sigma}_{i}^{k}(\tau)=K\left[\tilde{\varepsilon}_{i}(\tau), \tilde{E}_{1}^{k}(\tau), \tilde{E}_{2}^{k}(\tau), \tilde{G}_{12}^{k}(\tau), \tilde{v}_{12}^{k}(\tau), \theta^{k}, v^{k}\right]
$$

$\mathrm{L}, \mathrm{M}, \mathrm{R}, \mathrm{K}, \mathrm{P}$ are stochastic functional operators to be specified. According to this approach, current composite elastic properties, and therefore composite deformations and damage functions, are dependent on loading history. In order to integrate eq.(1), we have to calculate stochastic stress-strain field parameters in cells which depend on stochastic material properties and loading, as well as deformation history. The failure criterion (eq. 4) should be chosen to obtain probabilities of ply failure (damage functions). The stiffness reduction algorithm due to damage accumulation in plies (eq. 3) has to be specified.

\section{FAII,URE PROBABILITY CALCULATION}

Assume, that cell stochastic elastic characteristics $\tilde{E}_{l}^{k}, \tilde{E}_{2}^{k}, \tilde{G}_{12}^{k}, \tilde{v}_{12}^{k}$ are statistically independent and normally distributed random numbers. They can be described using mathematical mean values $\bar{E}_{1}^{k}, \bar{E}_{2}^{k}, \bar{G}_{12}^{k}, \bar{v}_{12}^{k}$ and dispersions $D_{E_{1}^{k},} D_{E_{2}^{k},} D_{G_{12}^{k}}, D_{v_{12}^{k}}$. Mathematical mean value and dispersion of a function of independent random variables $\tilde{y}=f\left(\tilde{x}_{1}, \tilde{x}_{2}, \ldots, \tilde{x}_{n}\right)$ can be evaluated as follows: 


$$
\begin{aligned}
& \bar{y}=f\left(\bar{x}_{1}, \bar{x}_{2}, \ldots, \bar{x}_{n}\right) \\
& D_{y}=\sum_{i=1}^{n}\left(\frac{\partial y}{\partial x_{i}}\right)^{2} D_{x_{1}}
\end{aligned}
$$

Stochastic cell strain response to the incremental laminate loading is calculated using normally distributed numbers properties. Mean values and dispersions of the following random variables are computed in sequence (see $[14,15]$ for some of the formulas involved):

(i) cell current technical elastic characteristics

(ii) cell stiffness coefficients in material axes

(iii) cell stiffness coefficients in laminate axes

(iv) sublaminas stiffness coefficients

(v) laminate stiffness coefficients

(vi) laminate compliance coefficients

(vii) laminate strain increments

(viii) total laminate strains

(ix) sublaminas stresses

(x) cell strains in laminate axes

(xi) cell strains in material axes

Cell properties averaging procedure according to weave parameter $v$ (iv), and classical lamination theory are used in the calculation. Procedure for current elastic properties estimation (i) is described below.

Maximal strain criterior is applied to calculate the failure probability of individual cells. We will describe damage criterion for deterministic and stochastic failure strains. In case of deterministic failure strains, each cell is assigned limiting failure strains in tension and compression, $\varepsilon_{i}^{\prime k}, \varepsilon^{\prime \prime}{ }_{i}^{k}$, respecti; ${ }^{\prime}$ ly. These limiting values are the same in magnitude for shear strain. In case of stochastic failure strains these are normal distributions. The probability of $\tilde{\varepsilon}_{i}^{k}$ to exceed deterministic border $\varepsilon_{i}^{\prime k}$ is simply the area under differential distribution of $\tilde{\varepsilon}_{i}^{k}$ on the right side of the upper bound $\varepsilon_{i}^{\prime k}$, and can be calculated from integral distribution $F_{\varepsilon_{i}^{k}}$ of ply strain by using eq.(7).

$$
r_{i}^{k}=1-F_{\varepsilon_{i}^{k}}\left(\varepsilon_{i}^{\prime k}\right)
$$

For stochastic failure strains ${\tilde{\varepsilon^{\prime}}}^{\prime}{ }_{i}$, probability $\tilde{\varepsilon}_{i}^{k}$ to exceed $\tilde{\varepsilon}^{\prime}{ }_{i}^{k}$ can be calculated as probability of random difference $\tilde{\varepsilon}_{i}^{k}-\tilde{\varepsilon}_{i}^{k}$ to be negative.

$$
r_{i}^{k}=F_{\varepsilon_{i}^{\prime k}-\varepsilon_{i}^{k}}(0)
$$

Parameters of integral distribution $F_{\varepsilon_{i}^{\prime}-\varepsilon_{i}^{k}}$ can be calculated as

$$
\begin{gathered}
\overline{\bar{\varepsilon}^{\prime k}{ }_{i}-\varepsilon_{i}^{k}}=\bar{\varepsilon}^{\prime k}{ }_{i}-\bar{\varepsilon}_{i}^{k} \\
D_{\varepsilon^{\prime k}{ }_{i}-\varepsilon_{i}^{k}}=D_{\varepsilon^{\prime k}{ }_{i}}+D_{\varepsilon_{i}^{k}}
\end{gathered}
$$


Taking into account both upper and lower bounds $\varepsilon_{i}^{\prime k}$ and $\varepsilon^{\prime \prime}{ }_{i}$, and assuming that strain field excursions beyond the two bounds are statistically independent events, the following expressions for $r_{i}^{k}$ can be derived

(a) deterministic bounds $\varepsilon_{i}^{\prime k}, \varepsilon^{\prime \prime}{ }_{i}^{k}$

$$
r_{i}^{k}=1-F_{\varepsilon_{i}^{k}}\left(\varepsilon_{i}^{\prime k}\right)+F_{\varepsilon_{i}^{k}}\left(\varepsilon^{\prime \prime}{ }_{i}^{k}\right)
$$

(b) random bounds $\tilde{\varepsilon}{ }^{k}, \tilde{\varepsilon}^{\prime \prime}{ }_{i}$

$$
r_{i}^{k}=F_{\varepsilon_{i}^{*}, \varepsilon_{i}^{k}}(0)+F_{\varepsilon_{i}^{k}-\varepsilon^{k}{ }_{i}^{*}}(0)
$$

It is easy to show that second case is reduced to first one when $D_{\varepsilon^{\prime k} i_{i}}=D_{\varepsilon^{\prime \prime}{ }_{i}^{k}}=0$. Thus, damage state of each cell is described by three damage parameters $r_{i}^{k}=\left\{r_{1}^{k}, r_{2}^{k}, r_{12}^{k}\right\}$. Note, that according to the developed model damage state in cells depends on weave of textile reinforcement and for $v \neq 0.5$, cells with the same orientation in top and bottom sublaminas, which have identical initial elastic characteristics, may become fairly distinct due to damage. Hence, each ply of textile laminate in the proposed model is composed of cells of four different types.

\section{EFFECT OF DAMAGE ON CELL PROPERTIES: MESOVOLUME CONCEPT}

In order to use the information about the failure probability we need to specify how is it realized in the composite, i. e. when and how much damages of different types do emerge and how do they effect the current mechanical properties of composite. Assume that there are three types of damages related to three in-plane deformations in each cell $\varepsilon_{i}^{k}=\left[\varepsilon_{11}^{k}, \varepsilon_{22}^{k}, \gamma_{12}^{k}\right]$. Failure type due to $\varepsilon_{11}^{k}$ exceeding the critical bound can be associated with fiber breakage, and those due to $\varepsilon_{22}^{k}$ and $\gamma_{12}^{k}$ - with matrix cracking in transverse to fiber direction and in shear, respectively.

Assume that each cell consists of certain number of mesovolumes. The mesovolume has to be structurally homogeneous, i.e. to contain sufficient amount of reinforcing fibers to be considered as a continuum, and at the same time it has to be comparatively small in order to satisfy the condition of stochastic homogeneity of stress and strain fields inside its envelope. Initial random characteristics of cells are equal to respective mesovolumes characteristics. Assume that each of the mesovolumes can be either perfect or broken in any of three ways, namely in fiber direction, in transverse direction and in shear. Therefore, we can suppose that at every current state of loading, the relative numbers of broken mesovolumes in cells for each type of failure are proportional to probabilities of failure $r_{i}^{k}$. In the limiting case of the infinite number of mesovolumes in cell the proportionality becomes equality. So, we can interpret the numbers $r_{i}^{k}=\left[r_{1}^{k}, r_{2}^{k}, r_{12}^{k}\right]$ as relative counts of broken meso-elements of three types in each cell.

Accumulation of damages in cells causes stiffness reduction of laminate and stress redistribution around the microcracks and between the cells and plies. This process is very complicated and modeling damage accumulation using micro-level consideration is, in general, not amenable to analytical studies. Probabilistic imitation approach [5] has lead to certain success in the area but it is computationally extensive and needs many simplifying assumptions to be 
made as well.

It is assumed in this paper that meso-element failure in certain direction causes deterioration of properties in the same direction. Failure of $r_{i}^{k}$ fraction of all meso-elements in i-th direction of $k$-th cell will result in reduction of cell elastic modulus in this direction. It is proposed that mathematical mean values of cell elastic moduli decrease with damage accumulation according to following hypothesis,

$$
\begin{aligned}
\bar{E}_{1}^{k}(t)=\bar{E}_{1_{0}}^{k}\left(1-r_{11}^{k}(t)\right) \quad \bar{E}_{2}^{k}(t)=\bar{E}_{2_{0}}^{k}\left(1-r_{22}^{k}(t)\right) \\
\bar{G}_{12}^{k}(t)=\bar{G}_{12_{0}}^{k}\left(1-r_{12}^{k}(t)\right) \quad=\bar{v}_{12}^{k}(t)=\bar{v}_{12_{0}}^{k}\left(1-r_{11}^{k}(t)\right)
\end{aligned}
$$

but relative standard deviations remain constant

$$
\frac{\sqrt{D_{E_{1}^{k}(t)}}}{\bar{E}_{1}^{k}(t)}=\frac{\sqrt{D_{E_{1}^{k}}}}{\bar{E}_{1_{0}}^{k}} \quad \frac{\sqrt{D_{E_{2}^{k}(t)}}}{\bar{E}_{2}^{k}(t)}=\frac{\sqrt{D_{E_{2_{0}}^{k}}}}{\bar{E}_{2_{0}}^{k}} \quad \frac{\sqrt{D_{G_{12}^{k}(t)}}}{\bar{G}_{12}^{k}(t)}=\frac{\sqrt{D_{G_{12_{0}}^{k}}}}{\bar{G}_{12_{0}}^{k}} \quad \frac{\sqrt{D_{v_{12}^{k}(t)}}}{\bar{v}_{12}^{k}(t)}=\frac{\sqrt{D_{v_{12_{0}}^{k}}}}{\bar{v}_{120}^{k}}
$$

According to this algorithm damage accumulation in cell during laminate loading evokes the shift of cell moduli distributions towards zero direction and narrows these distributions proportionally to stiffness decrease. Changes in statistical distributions of stiffness resulting from damage accumulation according to the proposed algorithm of stiffness reduction are illustrated in Figure 2.

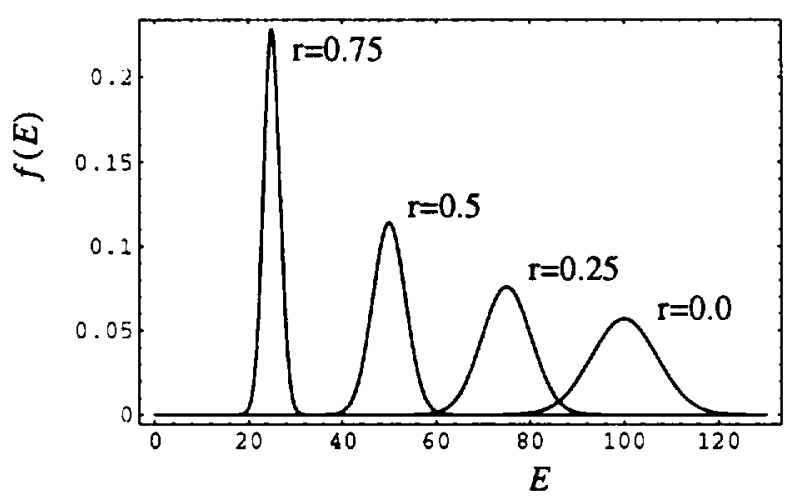

FIGURE 2. Stiffness distributions at various damage levels.

This simple approach does not take into consideration micromechanical phenomena like stress redistribution around the single cracks or cracks interaction, but does take into account gradual stiffness reduction due to damage accumulation in cells and stress redistribution between them.

\section{DISTRIBUTIE)NS OF CELL MATERIAL CHARACTERISTICS}

The basic feature of the proposed method is the consideration of stochastic nature of stressstrain field in the laminate due to stochastic stiffness and strength properties of laminate constituents. In addition, the concept of mesovolume as a building element for textile composites is introduced. Initial random properties of cells should be known to perform analysis. They can be obtained experimentally on small unidirectional specimens or can be calculated using some appropriate micromechanical models. In textile laminate, the scatter of angle of cell orientation 
in ply should be taken into account. Experimental inspection of specimens of fabric laminates shows that scatter of orientation depends essentially on the weave type and manufacturing technique.

Experimental statistical data for Kevlar/epoxy unidirectional composite presented in reference [16] are used in the following analysis. The random elastic and strength (critical strain bounds) characteristics are listed in Tables 1 and 2 (first line corresponds to zero deviation of angle). The standard deviations of random characteristics were calculated from their $95 \%$ confidential intervals given in [16], using well known statistical formulas.

Effect of scatter of orientation on distribution of cell properties was analyzed using the Monte-Carlo method. First, properties of unidirectional material and angle orientation were chosen at random according to their distributions. Then cell properties were calculated using rotational dependencies of the elastic and strength characteristics. Sets of several thousand samples were computed for each angle deviation value. Resulting mean values and standard deviations of variables are shown in Tables 1,2 . Some distributions of material characteristics obtained at $5 \mathrm{deg}$ orientation deviation are shown in Figures 3,4.
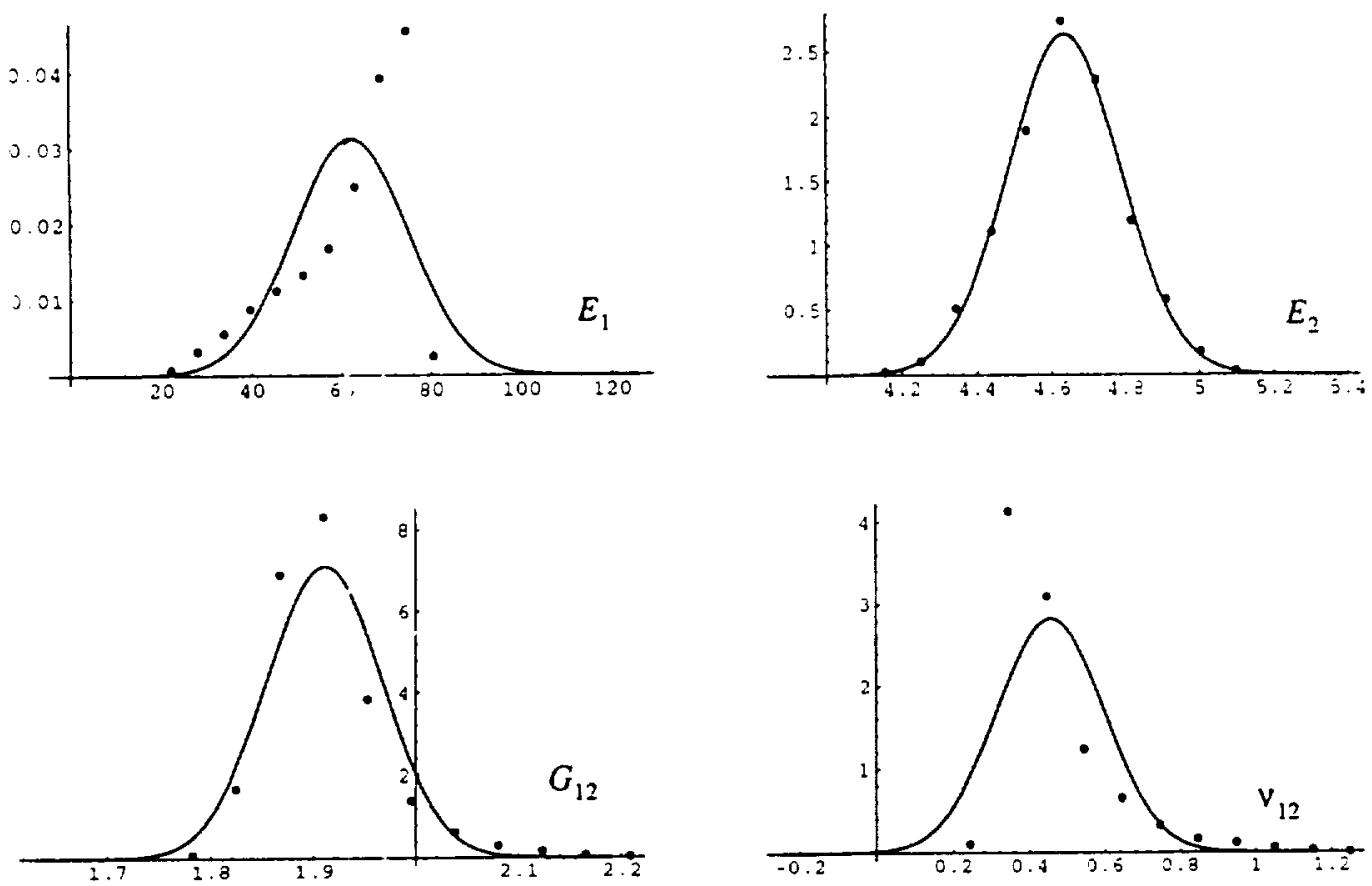

FIGURE 3. Calculated distriliutions of cell elastic characteristics (points) and their normal approximations (lines)

Analysis shows that scatter of orientation influences considerably the distributions of material properties. The strongest effect is observed for modulus $E_{1}$ and Poisson's ratio $v_{12}$ of the material. The increase of scatter of cell orientation causes decrease in tensile stiffness and slight increase in shear stiffness and failure strain of cell. In all cases, essential increase in deviation of material parameters is observed. Shape analysis of the resulting distributions shows that at larger deviations, some of them become significantly non-Gaussian. The reason for that is an extremal dependence of material properties on orientation angle around zero orientation. Fig- 
TABLE 1. Mean Value and Deviation of Cell Elastic Characteristics for Different Orientation Scatter

\begin{tabular}{|lllll|}
\hline $\begin{array}{l}\text { Angle St. } \\
\text { Deviation }\end{array}$ & $E_{1}$ & $E_{2}$ & $G_{12}$ & $v_{12}$ \\
$\operatorname{deg}$ & $\mathrm{GPa}$ & $\mathrm{GPa}$ & $\mathrm{GPa}$ & \\
\hline & & & & \\
\hline 0 & & & & \\
1 & $74.9 / 2.22$ & $4.65 / 0.16$ & $1.88 / 0.03$ & $0.35 / 0.031$ \\
2 & $74.0 / 2.48$ & $4.65 / 0.16$ & $1.88 / 0.03$ & $0.35 / 0.032$ \\
5 & $71.9 / 4.28$ & $4.65 / 0.16$ & $1.88 / 0.03$ & $0.36 / 0.039$ \\
10 & $62.0 / 12.9$ & $4.63 / 0.16$ & $1.91 / 0.05$ & $0.46 / 0.14$ \\
& $47.1 / 20.5$ & $4.60 / 0.16$ & $2.03 / 0.22$ & $0.71 / 0.37$ \\
\hline
\end{tabular}

* mean / standard deviation

TABLE 2. Mean Value and Deviation of Cell Failure Strains for Different Orientation Scatter

\begin{tabular}{|llllll|}
\hline Angle St. & $\varepsilon_{11}^{\prime}$ & $\varepsilon_{11}^{\prime \prime}$ & $\varepsilon_{22}^{\prime}$ & $\varepsilon_{22}^{\prime \prime}$ & $\gamma_{12}^{\prime}, \gamma_{12}^{\prime \prime}$ \\
Deviation & $C_{0}$ & $\%$ & $\%$ & $\%$ & $\%$ \\
$\operatorname{deg}$ & & & & & \\
\hline & & & & & \\
1 & $1.71 / .2$ & $-.478 / .024$ & $.283 / .016$ & $-1.41 / .11$ & $2.56 / .79$ \\
2 & $1.71 / .2$ & $-.478 / .024$ & $.284 / .016$ & $-1.41 / .11$ & $2.55 / .82$ \\
5 & $1.72 / .2$ & $-.478 / .024$ & $.283 / .015$ & $-1.41 / .11$ & $2.60 / .76$ \\
10 & $1.72 / .2$ & $-.482 / .026$ & $.285 / .016$ & $-1.42 / .11$ & $2.49 / .78$ \\
& $1.76 / .21$ & $-.494 / .035$ & $.292 /, 021$ & $-1.45 / .13$ & $2.35 / .92$ \\
\hline
\end{tabular}

* mean / standard deviation 
ure 5 shows the variation of elastic characteristcs of unidirectional composite with orientation. Figure 6 represents the variation of tensile and compressive deformations at failure with angle, and Figure 7 - the variation of shear failure strain. These extremal dependencies cause the shape change of distributions. In case of low initial scatter the distributions may become onesided.
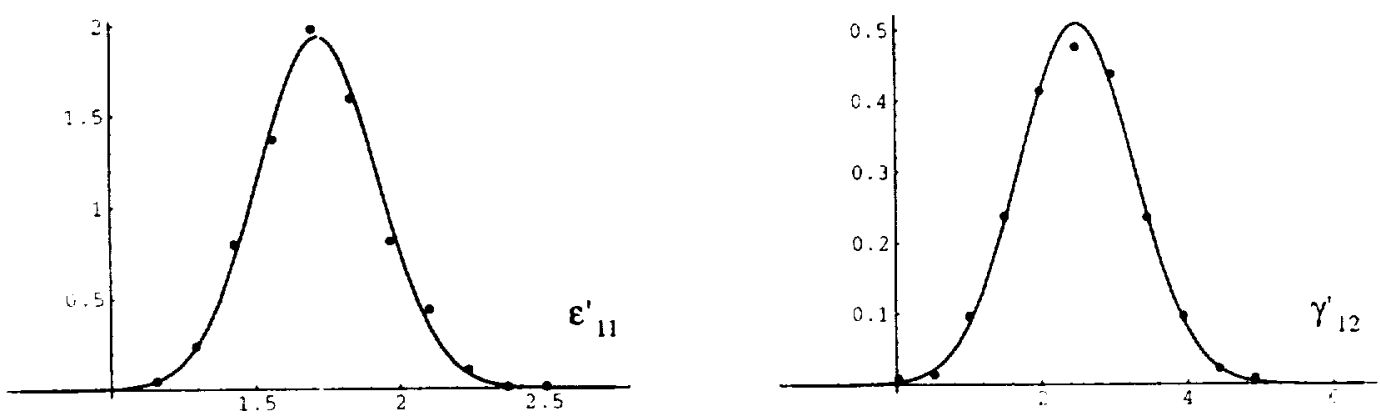

FIGURE 4. Calculated distributions of cell failure strains (points) and their normal approximations (lines).
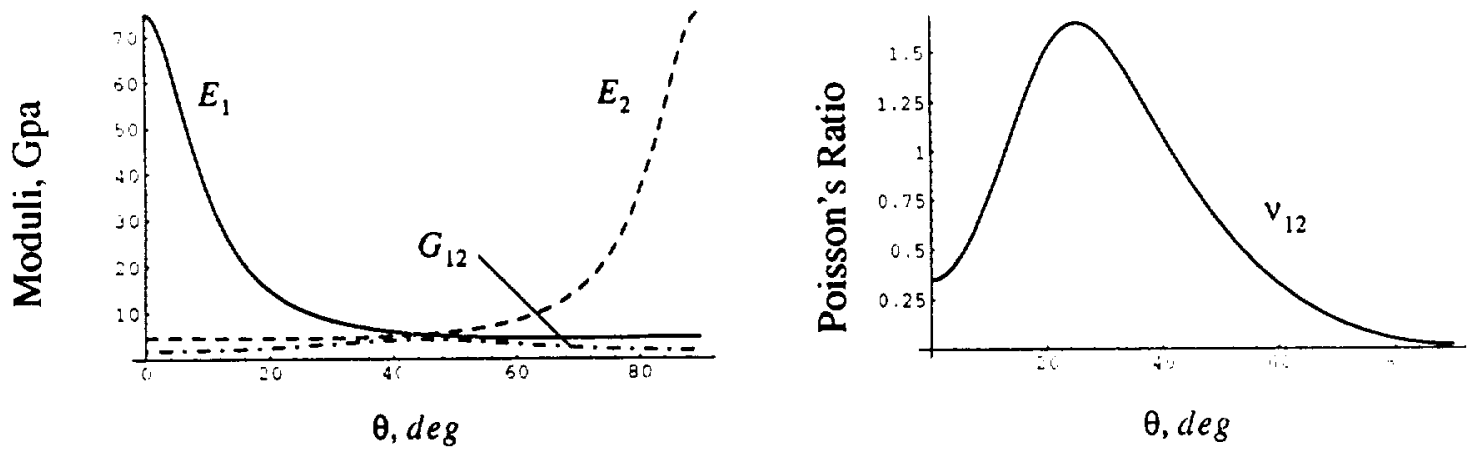

FIGURE 5. Variation of cell elastic characteristics with fiber orientation.

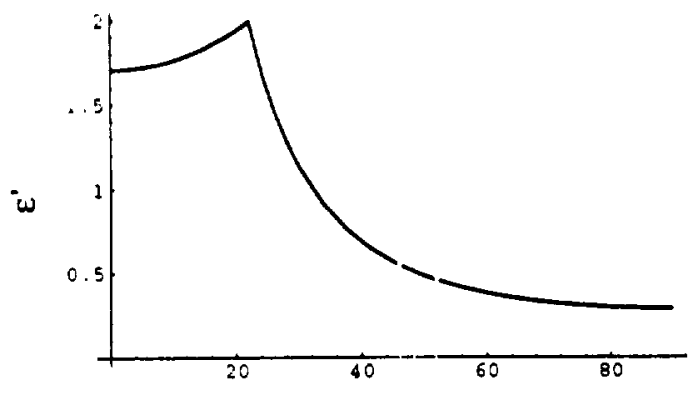

$\theta, d \in g$

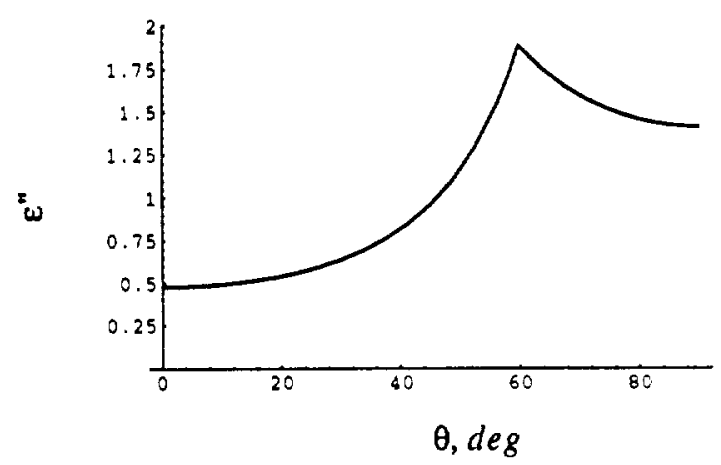

FIGURE 6. Variation of cell failure strains in tension $\varepsilon^{\prime}$ and compression $\varepsilon^{\prime \prime}$ with fiber orientation.

The performed calculation of third and fourth moments of distributions (i.e., coefficients 
of skewness and kwitosis) and their comparison with literature data [17] have shown that for the material under consideration the $5 \mathrm{deg}$ deviation of angle makes some of material distributions to deviate significantly from normal distribution. Further development of method is required to deal with these situations.
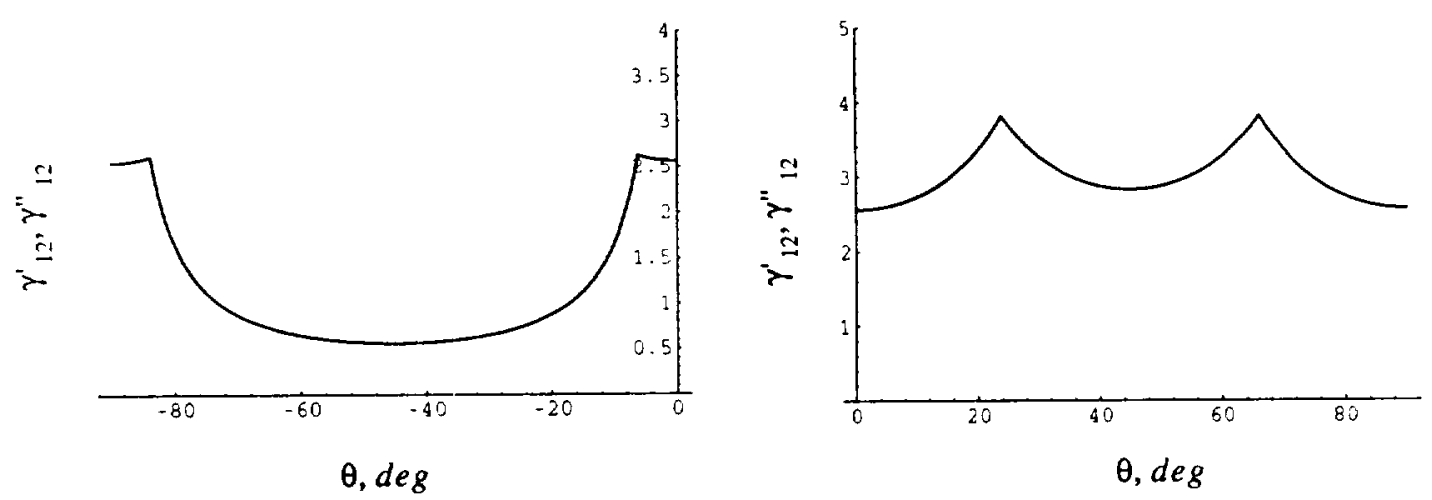

FIGURE 7. Variation of cell failure strain in shear with orientation.

\section{ILLUSTRATIVE EXAMPLE}

Computer code is developed for calculating laminate response under the in-plane loading. Incremental loading is set in the program according to given derivatives $\dot{\sigma}_{i}$ and equation (1) is integrated numerically. On each step of loading the damage functions in cells $r_{i}^{k}$ accumulated up to the previous step are used to calculate the current elastic properties of cells and laminate strain increments. It is assumed that complete failure of composite occurs when any of the current effective elastic moduli of laminate $\bar{E}_{1}, \bar{E}_{2}, \bar{G}_{12}$ becomes equal to zero (in program - equal to small number). The program is written in "Mathematica" and handles general case of arbitrary ply number, weave and arbitrary cell orientations in plies. Detailed information on damage functions and data on laminate strains and current effective properties are recorded to the data file on each loading step and plotted after completing the computation.

The capability of program is illustrated on an example of Kevlar/epoxy laminate consisting of identical textile reinforced plies. Orientation of cells in the laminate is [ \pm 45 ]. Characteristics of Kevlar/epoxy composite with 2 deg orientation deviation (Tables 1,2) are used as cell material properties. The following values of weave parameter $v$ were used in the analysis $-0.5,0.33$, 0.25 , and 0.11 , which correspond to plain weave, twill weave, 4 harness satin and 8 harness satin weave, respectively.

Calculated stress-strain diagrams due to shear loading and cumulative damage functions of the composite are shown in Figure 8. Continuous lines, dashed lines and dash-dot lines in the following figures correspond to 11,22 and 12 variables, respectively. Arrow points toward the lower values of $v$. Figure 9 shows the evolution of damages of different type in the laminate, obtained by averaging the damage functions over all cells. One can see that the more inhomogeneous distribution of cells between sublaminas is (lower $v$ ), the greater delay is observed for severe damage formation onset. The axial compressive deformations of laminate seen on the stress-strain diagrams (Figure 8) are due to damage induced anisotropy effect (see discussion in [15]). 

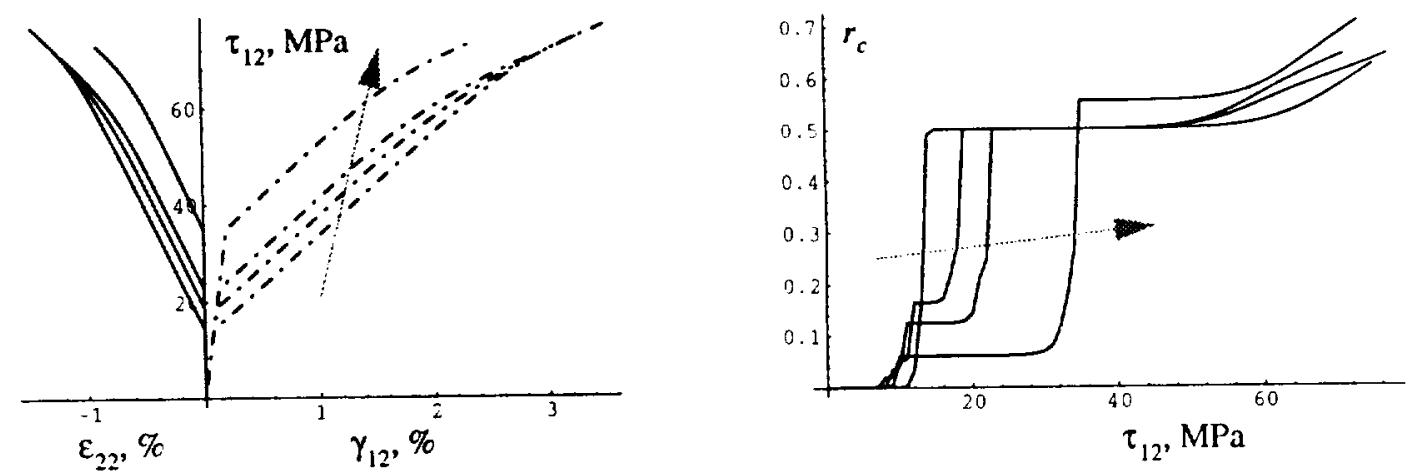

FIGURE 8. Stress-strain diagrams and cumulative damage functions of textile laminate at shear.
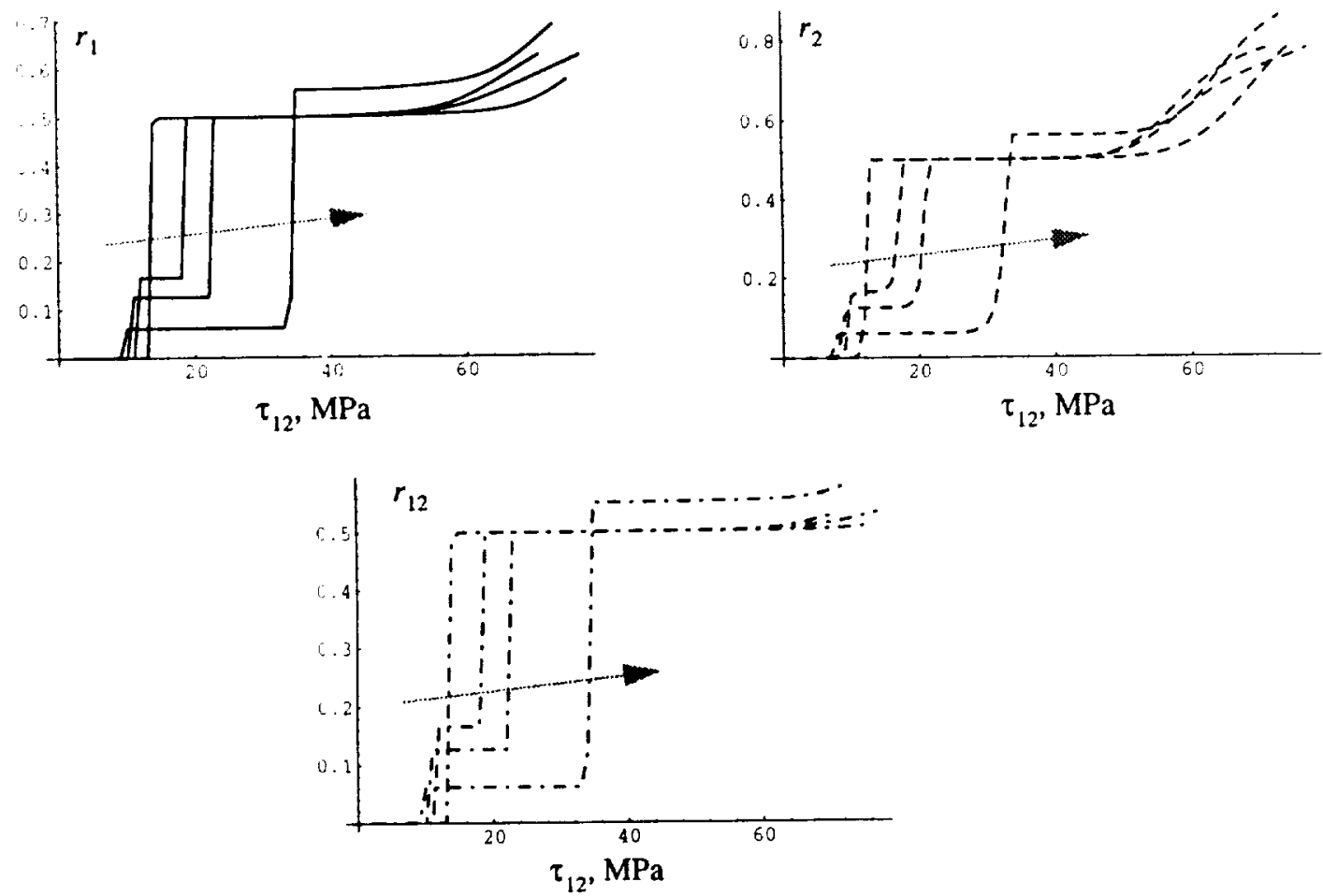

FIGURE 9. Average accumulation of damages of three types in textile laminate.

\section{CONCLUSIONS}

Damage evolution in textile laminates under the in-plane loading is discussed in this paper. A laminate model based on the special lamina subdivision is presented which predicts variation of properties with weave parameter. The damage accumulation in such a system is calculated based on the random function theory. Numerical algorithm and computer code for damage accumulation and deformation history prediction are developed. The effect of orientation scatter on the statistical distributions of cell material properties is discussed. 


\section{ACKNOWLEDGEMENTS}

Yu. A. Dzenis would like to thank his wife, Natasha, for patience.

\section{REFERENCES}

1. Ishikawa, T. and Chou, T.W., "One-Dimensional Micromechanical Analysis of Woven Fabric Composites", AIAA Journal, 1983, 21, pp.1714-1721.

2. Ishikawa, T. and Chou ,T.W., "Stiffness and Strength Behaviour of Woven Fabric Composites", Journal of Material Science, 1982, 17, pp.3211-3220.

3. Ishikawa, T. and Chou ,T.W., "In-Plane Thermal Expansion and Thermal Bending Coefficients of Fabric Composites", Journal of Composite Materials, 1983, 17, pp.92-104.

4. Bogdanovich, A.E. and Pasto" , C.M., "On the Structural Analysis of Textile Composites" -In: Topics in Composite Materials and Structures, AE-Vol 26, ASME,1992, pp.1391.

5. Ovchinskii A.S., "Fracture Processes in Composite Materials: Computer Imitation of Micro- and Macromechanisms", (in Russian). Nauka Publ. (Moscow), 1988.

6. Wagner H.D., "Statistical Concepts in the Study of Fracture Properties of Fibres and Composites" - In: Composite Materials Series. Vol. 6. Application of Fracture Mechanics to Composite Materials, K. Friedrich, ed., Elsevier, 1989, pp.39-77.

7. Argon A., "Statistical Aspects of Fracture" - In: Composite Materials. Vol. 5. Fracture and Fatigue, Acad. Press, 1974, pp. 166-205.

8. Bogdanovich, A.E. and Yushanov, S.P., "Buckling Analysis of Cylindrical Shells Having Random Field of Initial Imperfections Under Axial Dynamic Compression", Mechanics of Composite Materials (Riga), 1981, 5, pp. 821-831.

9. Bogdanovich, A.E. and Yushanov, S.P., "On the Calculation of Reliability Function of Anisotropic Shells by Using Technique of Seldom Outbursts of a Stochastic Vector Field Out of the Limiting Surface", Mechanics of Composite Materials (Riga), 1983, 1, pp. 8089.

10. Yushanov, S.P. and Bogdanovich, A.E. "Method of Reliability Calculation for Imperfect Laminated Cylindrical Shells with Account for Scatter of Composite Strength Characteristics", Mechanics of Composite Materials (Riga), 1986, 6, pp. 1043-1048.

11. Yushanov, S.P., "On Reliability Calculation of Laminated Composite Shells with Random Elastic and Strength Characteristics", Mechanics of Composite Materials (Riga), 1985, 1, pp. 87-96.

12. Yushanov, S.P., "Probabilistic Model of Composite Ply-by-Ply Failure and Reliability Calculation of Laminated Cylindrical Shells", Mechanics of Composite Materials (Riga), 1985,4 , pp. 642-652.

13. Bogdanovich, A.E. and Yushanov, S.P., "A Reliability Analysis of Laminated Composites and Cylindrical Shells" - Proc. of the Sixth Int. Conf. on Compos. Mater. and Second Europe. Conf. on Compos. Mater., London, 1987. Elsevier Appl. Sci. Publ., 1987, vol. 5, pp. 5.525-5.535. 
14. Dzenis Yu.A., Joshi S.P., Bogdanovich A.E., Damage Evolution Modeling in Orthotropic Laminated Composites - Proceedings of the 33rd AIAA/ASME/ASCE/AHS/ASC Structures, Structural Dynamics and Material Conference, April 13-15, 1992, Dallas. Part 5 , p.2887-2897.

15. Dzenis Yu.A., Joshi S.P., Bogdanovich A.E., Damage Evolution Analysis of Laminates Subjected to Random Quasistatic Loading - Proceedings of the 7th ASC Technical Conference on Composite Materials, October 13-15, 1992, University Park, Pennsylvania, p.1084-1093.

16. Clements, L.L. and Chiau, T.T., "Engineering Design Data for an Organic Fiber/Epoxy Composite", Composites, Vol. 8, No. 2, April 1977, pp. 87-92.

17. Pearson, E.S. and Hartley, H.O., "Biometrika Tables for Statisticians. Vol 1", Campridge, 1966. 